\title{
On sealing effects and oxygen dependence of non-recoverable intensity drops in short-chain-conjugated polymers
}

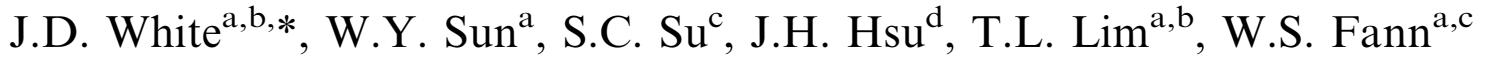 \\ ${ }^{a}$ Institute of Atomic \& Molecular Sciences, Academia Sinica, Taipei, Taiwan \\ ${ }^{\mathrm{b}}$ Centre for Highspeed and Broadband Networking, Multimedia University, 75450 Maleka, Malaysia \\ ${ }^{\mathrm{c}}$ Department of Physics, National Taiwan University, Taipei, Taiwan \\ d Department of Chemistry, National Taiwan University, Taipei, Taiwan
}

\begin{abstract}
The fluorescence time trace of persistent length single poly (2,5-dioctyloxy p-phenylene vinylene) (DOO-PPV) polymers spin-cast in an inert matrix is recorded under different sealing conditions - which effectively results in different levels of oxygen exposure. The frequency of non-recoverable intensity drops is seen to be dependent on oxygen concentration. Qualitative differences in published results for the photophysics of long-chain polymers and rod-like polymers are shown to be due to chain length differences and not due to sealing conditions. (C) 2002 Elsevier Science B.V. All rights reserved.
\end{abstract}

PACS: 80.33. cd

Keywords: Single molecule; Conjugated polymer; Photophysics; Fluorescence

Considerable insights into the photophysics and energy transfer within luminescent conjugated polymers can be gained by studying the fluorescence time trace of single polymers under constant excitation. Extensive studies have been done on members of the p-phenylene vinylene (PPV) family. While the fluorescence emission from a single polymer may follow a simple exponential decay [1]; it may also be punctuated by abrupt changes in intensity [2]. Differences in conformation [3], which may be traced back to the solvents

\footnotetext{
*Corresponding author. Centre for Highspeed and Broadband Networking, Multimedia University, 75450 Maleka, Malaysia. Tel.: +606-252-3037; fax: +606-231-6555.

E-mail addresses: whitejd@xiaotu.com (J.D. White), fann@gate.sinica.edu.tw (W.S. Fann).
}

used in the preparation of the thin film [3], have been proposed as the underlying cause. When abrupt changes are observed, emission is seen at only a few discrete intensities [3]. In contrast, in rod-like polymers, emission is seen at a number of discrete intensities, which are often multiples of a fundamental intensity [4]. While chain length and resulting conformation differences have been used by way of explanation, another possible explanation is the difference in sample-sealing techniques, as the solvents used are similar. In one case, sealing has been done in a nitrogen environment [5], while in the other aluminum coating has been used [2]. It is not at all clear whether the aluminum coating is affecting the results in ways other than reducing the access to oxygen (i.e. metal quenching). The purpose of this investigation is to 
determine whether the difference between longand short-chain behavior is merely the result of different sealing conditions.

Secondly, if qualitatively equivalent behavior is seen between the aluminum-coated samples and the dry nitrogen-sealed samples, these different sealing conditions present a way to investigate the effect of oxygen content on abrupt intensity changes. These abrupt changes in intensity can be divided into two distinct categories: nonrecoverable and recoverable intensity drops. In the first, the quenching is permanent while in the second the quenching is only temporary and emission shortly returns back to the original level. In this paper, we address the issue of nonrecoverable intensity drops and their dependence on oxygen concentration, leaving the second question to an additional work.

Pristine poly (2,5-dioctyloxy p-phenylene vinylene) (DOO-PPV) [6] was fractionalized by taking advantage of the limited solubility of this symmetrically substituted structure. For the purposes of this experiment, the fraction having molecular weight $\left(M_{n}\right) \sim 8.2 \mathrm{kDa}$ and polydispersity $(\delta=$ $\left.M_{w} / M_{n}\right)=2.23$ was extracted and used. This fraction was chosen in order to closely match the persistent length (polymer of $\sim 24$ monomers long) [7]. As, an exciton is expected to be delocalized over $\sim 6$ monomers [8], our sample polymer is expected to support $\sim 4$ excitons. It should be noted, however, that the distribution is quite asymmetric with a large distortion towards the longer polymer lengths so that polymers with more excitons active should also be observed. After dilution in an inert polystyrene matrix, the polymer was spin-cast onto a fused silica substrate. The resulting film thickness was $\sim 100 \mathrm{~nm}$. In order to investigate the effects of sealing conditions, three different sets of samples were investigated. The first set of samples was investigated directly with no attempts to protect the thin film from oxygen and humidity. The second set was placed in a nitrogen glove box for sealing. After a few hours nitrogen at low pressure, the slide glass was placed in a special holder designed to keep dry nitrogen in contact with the thin film. The final set was coated with a thin aluminum film after a few hours in a vacuum chamber. As well as allowing the effects of the aluminum film to be seen, these three sealing techniques provide progressively more effective oxygen exclusion.

The samples were then observed using a homemade confocal microscope [5]. A continuous linearly polarized TEM00 laser beam $(488 \mathrm{~nm})$ was focused by an oil immersion objective lens (Nikon Plan Fluo NA 1.3) through the fused silica slide glass and onto a single polymer. Fluorescence was collected in epifluorescence mode with an APD operating in photon counting mode. In general, data were taken using an open-loop tube piezoelectric controller (Fig. 1). However, due to the long lifetime of the aluminum-coated samples, a closed-loop configuration was found necessary to minimize drift in this case (Fig. 2).

In the case in which no particular efforts were made to protect sample from oxygen and humidity the average sample lifetime was in the range of a few seconds at the levels of excitation being used. However, despite the short lifetime, multiple step intensity drops were clearly observed $(\sim 4)$ in the fluorescence time trace. However, due to the short lifetime, only very rarely was an abrupt increase in

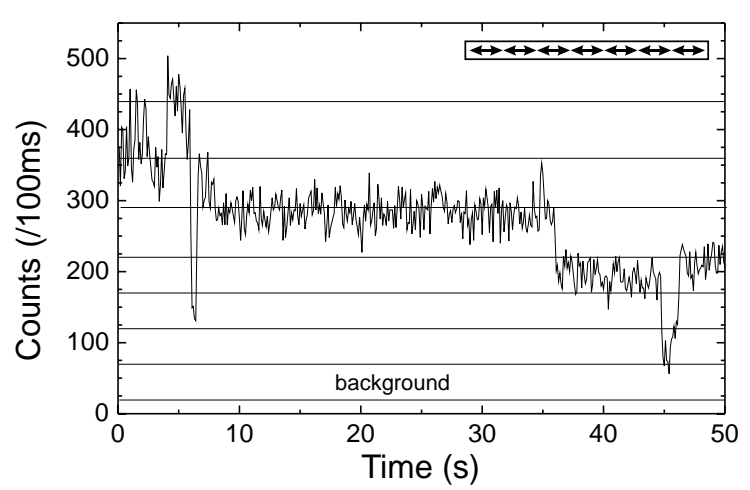

Fig. 1. First $50 \mathrm{~s}$ of a fluorescent time trace of a single polymer in which moderate efforts were made to protect the sample from exposure to oxygen and humidity (see text for details). The total lifetime of this polymer was $\sim 85 \mathrm{~s}$. Data were taken with an excitation irradiance of $200 \mathrm{~W} / \mathrm{cm}^{2}(300 \mathrm{~nm}$ spot size) and is displayed with an integration time constant of $100 \mathrm{~ms}$. The horizontal lines are slightly slanted to compensate to first order for drift (due to the use of an open-loop piezo). Inset shows a possible conformation that fits the experimental data. Horizontal lines illustrate the emission levels expected from this conformation. 


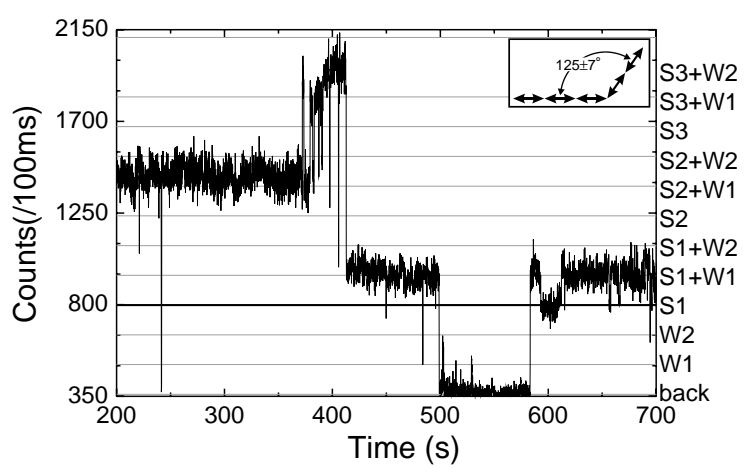

Fig. 2. $500 \mathrm{~s}$ of the fluorescence time trace of a single polymer in which the sample was sealed with aluminum (see text for details). The total lifetime of this polymer was $4200 \mathrm{~s}(70 \mathrm{~min})$. Data were taken with an excitation irradiance of $1100 \mathrm{~W} / \mathrm{cm}^{2}$ (assuming a $450 \mathrm{~nm}$ spot size) and is displayed with an integration time constant of $100 \mathrm{~ms}$. The inset illustrates a polymer conformation that fits the data. Assuming this conformation, the right axis shows the combination of emitting excitons that contribute to each emission level. For example, the label S2 + W1 indicates that two excitons aligned with the exciting radiation and one exciton at an angle with respect to the polarization of excitation light.

intensity observed. In other words, most intensity drops were non-recoverable. It should be noted that decreasing the excitation irradiance by two orders of magnitude resulted in an order of magnitude increase in sample lifetime. Unfortunately, at this level of excitation, the low $S / N$ ratio made data analysis difficult.

Fig. 1 presents the first $50 \mathrm{~s}$ of the fluorescent time trace for a single polymer having limited exposure to oxygen and moisture (in contact with dry nitrogen). In this case the fluorescence time trace is an order of magnitude longer (about $90 \mathrm{~s}$ ). Emission occurs at seven discrete intensities suggesting a model of seven aligned, independently absorbing and emitting excitons. Obvious in this time trace is the clear existence of not only nonrecoverable, but also recoverable intensity drops. For example, the drop at $6 \mathrm{~s}$ recovers $0.5 \mathrm{~s}$ later and that at $44 \mathrm{~s}$ recovers $1 \mathrm{~s}$ later.

Fig. 2 presents part of a typical fluorescent time trace for a polymer in a sample in which aluminum coating ( $\sim 200 \mathrm{~nm}$ thick) was used as an oxygen barrier. This polymer continued to emit for a total of $70 \mathrm{~min}$. For the sake of clarity, in this figure we have limited our presentation to a $500 \mathrm{~s}$ segment.
Even when looking in this window, the amount of data makes it difficult to determine by just looking at the graph whether changes in intensity are recoverable intensity drops or just noise. For example, on the main graph there is a sudden drop at $241 \mathrm{~s}$. It is only by expanding the time scale that we can state for sure that this is indeed a recoverable intensity drop to background level that lasts for $500 \mathrm{~ms}$. In this molecule, emission occurs at nine discrete intensities. The inset provides a conformation that explains this emission. Three excitons have their dipoles aligned closely with the polarization of the excitation laser, while a tetrahedral defect results in the remaining two excitons having a much lower absorption cross-section.

Taking these results as a whole, we can now make some conclusions relating to the originally posed questions. With regard to the first question, "Does the aluminum coating affect the results in ways other than in providing a barrier?" the answer seems to be "No". Comparing Figs. 1 and 2 and ignoring the time scale, the traces are remarkably similar. In both cases, multiple steps are observed (in contrast to the long-chain polymer results). In both cases, both recoverable and non-recoverable intensity drops are observed. Thus, we conclude the differences in behavior between long- [1-3] and short-chain polymers [4,5] are due to the difference in chain length and not the sealing conditions. Aluminum coating is thus verified to be an effective way of limiting exposure to oxygen without seriously affecting the single polymer photophysics.

Having verified that the aluminum coating does not qualitatively affect the results, let us turn our focus on the quantitative effects of the aluminum coating both with respect to the intensity and time scales. Under similar sealing conditions, the intensity of the fluorescent time trace is directly proportional to the excitation irradiance. Comparing Figs. 1 and 2, one sees that there is a difference in excitation irradiance of a factor of 5.5. Thus, for the same polymer, one would expect that emission in the aluminum sealed case should be 5.5 higher than the nitrogen-sealed case. Looking at the maximum emission level, it appears that the emission level only increases by a factor of 4 . 
One might conclude that the effect of the aluminum coating is a $20 \%$ reduction in emitted light intensity. However, this would not be an accurate conclusion as the above argument assumes that one is looking at the same polymer. The emission intensity is a function of: (1) the number of active conjugated segments in the polymer, (2) the conformation (linear or kinked), and (3) the alignment of the dipoles relative to the excitation irradiance (linearly polarized light is being used in this experiment). A meaningful comparison requires the consideration of the ensemble average fundamental step size. Our data to date only allows us to put bounds on the effects of aluminum coating on the observed intensities. We can conclude that: (1) aluminum coating does not result in an increase in observed intensity (no mirror effect or else any mirror effect is more compensated for by another mechanism) and (2) the drop in observed intensity is less than a factor of 5. This drop in intensity may be the result of quenching due to a sample-metal interaction. The mechanism may be resonance energy transfer from polymer to metal - operative at the length scale of $\sim 10 \mathrm{~nm}$. Since the film used in the experiments is thin, this effect might be important.

In conclusion, this paper has shown that the qualitative differences in behavior between longand short-chain polymers are not due to the use of aluminum in the coating, but rather due to differences in the chain length. The effect of aluminum coating is seen rather to be quantita- tive - reducing the observed emission intensity and drastically increasing the overall lifetime of the fluorescence time trace due to the effective exclusion of oxygen. The strong oxygen-concentrationdependent behavior of non-recoverable intensity drops is seen to be as a result of oxygen-dependent bleaching of these individual excitons.

\section{Acknowledgements}

The authors acknowledge the assistance of Y.C. Lee in preparing the experiments and financial support from the National Science Foundation and Ministry of Education, Taiwan, ROC.

In Jesus name.

\section{References}

[1] T. Huser, M. Yan, L.J. Rothberg, PNAS 97 (2000) 11187.

[2] J. Yu, D. Hu, P.F. Barbara, Science 289 (2000) 1327.

[3] D. Hu, J. Yu, K. Wong, B. Bagchi, P.J. Rossky, P.F. Barbara, Nature 405 (2000) 1030.

[4] J.D. White, et al., Chem. Phys. Lett. 238 (2001) 263.

[5] J.D. White, et al., J. Chem. Phys. 114 (2001) 3848.

[6] S.H. Askari, S.D. Rughoophth, F. Wudl, Synth. Methods 29 (1989) E129-E134.

[7] C.L. Gettinger, A.J. Heeger, J.M. Drake, D.J. Pine, J. Chem. Phys. 101 (1994) 1673.

[8] M. Yan, L.J. Rothbers, F. Papadimitrakopoulos, M.E. Galvin, T.M. Miller, Phys. Rev. Lett. 73 (1994) 744. 